Jurnal Matematika Thales (JMT): 2019

Vol. 01-01

\title{
ON LEFT QUASI-EXACT SEQUENCES
}

(SEPUTAR BARISAN QUASI EKSAK KIRI)

\author{
SITI MUAWANAH, INDAH EMILIA WIJAYANTI
}

\begin{abstract}
The aim of this paper is to bring together the properties of quasi-exact sequences and homomorphism functors. It is of interest to know whether the left quasi-exact sequences have some interesting properties related to the functors and vice versa. Moreover, we also define the dualization of left quasi-exact sequences, i.e. right quasi-coexact sequences. Our proofs show that the quasi-exactness of sequences is preserved by any homomorphism functors.

Keywords: Exact sequences, quasi-exact sequences, left quasi-exact sequences, right quasi-coexact sequences, homomorphism functors.
\end{abstract}

\begin{abstract}
Abstrak. Salah satu sifat barisan modul-modul dan homomorfisma adalah keeksakan barisan tersebut terkait dengan sifat fungtor homomorfisma yang dikenakan padanya. Barisan quasi eksak kiri merupakan perumuman barisan eksak kiri. Dalam penelitian ini dilihat hubungan barisan quasi eksak kiri dengan fungtor homomorfisma. Selanjutnya didefinisikan pula dualisasinya yaitu barisan quasi koeksak kanan. Penelitian ini membuktikan bahwa sifat barisan quasi eksak kiri maupun kanan dipertahankan oleh fungtor homomorfisma.

Kata-kata kunci: Barisan eksak, barisan quasi eksak, barisan quasi eksak kiri, barisan quasi eksak kanan, fungtor homomorfisma.
\end{abstract}

\section{INTRODUCTION}

Exact sequences play important roles in module theory and homology. Some notions such as injectivity and projectivity of modules, generators and subgenerators of modules, have been defined and analyzed by exact sequence approach (see [6]). Generalizing exact sequences to quasi-exact sequences gives possibilities to generalized some related notions which are defined by exact sequences approach. In this work, we continue to observe further properties of quasi-exact sequences introduced by Anvariyeh and Davvaz (2] and [3]). We will restrict our discussion to left quasi-exact sequences as a generalization of left exact sequences. It is our

2010 Mathematics Subject Classification: 16D10 
purpose to study the applications of homomorphism functors to left quasi-exact sequences. It is of interest to know whether the left quasi-exact sequences have some interesting properties related to the functors and vice versa. We also define the dualization of left quasi-exact sequences, i.e. right quasi-coexact sequences.

Let $R$ be an associative ring with unit and the modules mean left unital $R$ modules. We consider some well known exactness of sequences. A more complete explanation may be obtained by Adkins and Weintarub [1] or Wisbauer 6]. A sequence of $R$-modules and $R$-homomorphisms

$$
\ldots \stackrel{f_{i-1}}{\longrightarrow} A_{i-1} \stackrel{f_{i}}{\longrightarrow} A_{i} \stackrel{f_{i+1}}{\longrightarrow} A_{i+1} \stackrel{f_{i+2}}{\longrightarrow} \ldots
$$

is called an exact sequence in $A_{i}$ if $\operatorname{Im}\left(f_{i}\right)=\operatorname{Ker}\left(f_{i+1}\right)$. This sequence is called an exact sequence if it is exact in $A_{i}$ for every $i$. For a special case, if the sequence

$$
0 \longrightarrow A \stackrel{f}{\longrightarrow} B \stackrel{g}{\longrightarrow} C \stackrel{h}{\longrightarrow} D
$$

is exact, then $f$ is injective, $\operatorname{Im}(f)=\operatorname{Ker}(g), \operatorname{Im}(g)=\operatorname{Ker}(h)$, and we call it a left exact sequence. On the other hand, if the sequence

$$
A \stackrel{f}{\longrightarrow} B \stackrel{g}{\longrightarrow} C \stackrel{h}{\longrightarrow} D \longrightarrow 0
$$

is exact, then $\operatorname{Im}(f)=\operatorname{Ker}(g), \operatorname{Im}(g)=\operatorname{Ker}(h), h$ is surjective, and we call it a right exact sequence.

Anvariyeh and Davvaz in [5] introduced a quasi-exact sequence which is a generalization of exact sequences. A sequence of $R$-modules and $R$-homomorphisms $A \stackrel{f}{\longrightarrow} B \stackrel{g}{\longrightarrow} C$ is quasi-exact in $B$ or $U$-exact in $B$ if there exists a submodule $U$ in $C$ such that $\operatorname{Im}(f)=g^{-1}(U)$. A sequence $0 \longrightarrow A \stackrel{f}{\longrightarrow} B \stackrel{g}{\longrightarrow} C \longrightarrow 0$ is called a short $U$-exact sequence or short quasi-exact sequence if $f$ is injective, $g$ is surjective and $\operatorname{Im}(f)=g^{-1}(U)$ for some submodule $U$ in $C$ [3]. Anvariyeh and Davvaz studied some properties of short $U$-exact sequence such as the generalization of Five Lemma, ascending and descending chain of modules in sequences [5. Moreover, Anvariyeh and Davvaz observed the dual of quasi-exact sequences and $U$-split sequences [2]. The generalization of Snake Lemma was observed by Davvaz and Solt in quasi-exact sequences and they gave some results in concepts of generalization in algebra homology [4]. Furthermore, Anvariyeh and Davvaz investigated some properties of finitely generated modules, essential submodules, small submodules and Schanuel Lemma in a short $U$-exact sequence [3].

Consider now the following sequence:

$$
0 \longrightarrow A \stackrel{f}{\longrightarrow} B \stackrel{g}{\longrightarrow} C \stackrel{h}{\longrightarrow} D
$$

which is $U_{B}$-exact in $A, U_{C}$-exact in $B$, and $U_{D}$-exact di $C$, for some $U_{B} \subseteq B$, $U_{C} \subseteq C$ and $U_{D} \subseteq D$. Based on the definition of quasi-exact sequence, it yields $0=$ 
$f^{-1}\left(U_{B}\right)$. It is clear that $\operatorname{Ker}(f) \subseteq f^{-1}\left(U_{B}\right)=0$, so $\operatorname{Ker}(f)=0$ and moreover $f$ is injective. We call such sequence as a left quasi-exact sequence. If in the Sequence (1.1) we put $D=0$, then $U_{D}=0$, so $g$ is surjective and the Sequence (1.1) is a short $U_{C}$-exact sequence. Hence the short quasi-exact sequence is a special case of a left quasi-exact sequence.

Now we recall the definition of $V$-coexact sequences as a dualization of quasiexact sequences.

Definition 1.1. 3] A sequence of R-modules $A \stackrel{f}{\longrightarrow} B \stackrel{g}{\longrightarrow} C$ is called $V$ coexact in $B$ if there exists a submodule $V$ of $A$ such that $f(V)=\operatorname{Ker}(g)$.

Any $U$-exact sequence $A \stackrel{f}{\longrightarrow} B \stackrel{g}{\longrightarrow} C$ is a $\operatorname{Ker}(g f)$-coexact sequence. Conversely, any $V$-coexact sequence $A \stackrel{f}{\longrightarrow} B \stackrel{g}{\longrightarrow} C$ is an $\operatorname{Im}(g f)$-exact sequence. Moreover, a sequence

$$
\ldots \stackrel{f_{i-1}}{\longrightarrow} A_{i-1} \stackrel{f_{i}}{\longrightarrow} A_{i} \stackrel{f_{i+1}}{\longrightarrow} A_{i+1} \stackrel{f_{i+2}}{\longrightarrow} \ldots
$$

is called dual quasi-exact if it is $V_{i-1}$-coexact in $A_{i}$ for every $i$, where $V_{i-1}$ is a submodule of $A_{i-1}$.

As a dual of quasi-exact sequence, every short $U$-exact sequence

$$
0 \longrightarrow A \stackrel{f}{\longrightarrow} B \stackrel{g}{\longrightarrow} C \longrightarrow 0
$$

is a short $\operatorname{Ker}(g f)$-coexact sequence. Conversely, every short $V$-coexact sequence

$$
0 \longrightarrow A \stackrel{f}{\longrightarrow} B \stackrel{g}{\longrightarrow} C \longrightarrow 0
$$

is a short $\operatorname{Im}(g f)$-exact sequence. Hence, we can apply some properties of a short $U$-exact sequence for a short $V$-coexact sequence.

In the next section we discuss the notions of left quasi-exact sequences and the implications of applying hom-functor to the sequence. Our main results will be found in Proposition 2.5 and Proposition 2.6. The last section presents an introduction of right quasi-coexact sequences as the dual of left quasi-exact sequences.

\section{LEFT QUASI-EXACT SEQUENCES}

A sequence $0 \longrightarrow A_{1} \stackrel{f_{1}}{\longrightarrow} A_{2} \stackrel{f_{2}}{\longrightarrow} A_{3} \stackrel{f_{3}}{\longrightarrow} A_{4} \stackrel{f_{4}}{\longrightarrow} \ldots$ is quasi-exact if $f_{1}$ injective and it is $U_{i+1}$-exact in $A_{i}$ for every $i \geqslant 2$, where $U_{i+1}$ is a submodule of $A_{i+1}$. Now we give the definition of a left quasi-exact sequence.

Definition 2.1. A sequence $0 \longrightarrow A \stackrel{f}{\longrightarrow} B \stackrel{g}{\longrightarrow} C \stackrel{h}{\longrightarrow} D$ is called a left quasi-exact sequence if $f$ is injective and it is $U_{C}$-exact in $B$ and $U_{D}$-exact in $C$ for some $U_{C}$ submodule of $C$ and $U_{D}$ submodule of $D$. 
Example 2.2. The sequence of $\mathbb{Z}$-modules $0 \longrightarrow \mathbb{Z}_{2} \stackrel{i_{1}}{\longrightarrow} \mathbb{Z}_{4} \stackrel{i_{2}}{\longrightarrow} \mathbb{Z}_{8} \stackrel{i_{3}}{\longrightarrow} \mathbb{Z}_{16}$ is a left quasi-exact sequence, where $i_{1}(\bar{a})=2 \bar{a}$ for all $\bar{a} \in \mathbb{Z}_{2}, i_{2}(\bar{b})=2 \bar{b}$ for all $\bar{b} \in \mathbb{Z}_{4}$, and $i_{3}(\bar{c})=2 \bar{c}$ for all $\bar{c} \in \mathbb{Z}_{8}$.

Moreover, a sequence $0 \longrightarrow A \stackrel{f}{\longrightarrow} B \stackrel{g}{\longrightarrow} C \stackrel{h}{\longrightarrow} D$ is left quasi-exact if $f$ is injective, $\operatorname{Im}(f)=g^{-1}\left(U_{C}\right)$, and $\operatorname{Im}(g)=h^{-1}\left(U_{D}\right)$ for some submodule $U_{C}$ in $C$ and $U_{D}$ in $D$. We get a special case when $D=0$ as we consider in the following equivalence conditions.

Lemma 2.3. A sequence

$$
0 \longrightarrow A \stackrel{f}{\longrightarrow} B \stackrel{g}{\longrightarrow} C \stackrel{h}{\longrightarrow} D
$$

is a left quasi-exact sequence which is D-exact in $C$ if and only if the sequence

$$
0 \longrightarrow \text { ㄴ } \stackrel{f}{\longrightarrow} B \stackrel{g}{\longrightarrow} C \stackrel{h}{\longrightarrow} 0
$$

is short $U_{C}$-exact for a submodule $U_{C}$ in $C$.

Proof. $(\Rightarrow)$ Based on the assumption $f$ is injective, $\operatorname{Im}(f)=g^{-1}\left(U_{C}\right)$ for some $U_{C}$ in $C$ and $\operatorname{Im}(g)=h^{-1}(D)$. It is clear that $h^{-1}(D)=C$, so $\operatorname{Im}(g)=C$ and $g$ is surjective. Then we form the Sequence (2.2) where $f$ is injective, $g$ is surjective, and $\operatorname{Im}(f)=g^{-1}\left(U_{C}\right)$ for some submodule $U_{C}$ di $C$. Hence the sequence is short $U_{C^{-} \text {-exact. }}$

$(\Leftarrow)$ Based on the assumption $f$ is injective, $g$ is surjective and $\operatorname{Im}(f)=g^{-1}\left(U_{C}\right)$ for a submodule $U_{C}$ in $C$, so $\operatorname{Im}(g)=C$. For any $R$-homomorphism $h: C \longrightarrow D$, $h^{-1}(D)=C=\operatorname{Im}(g)$. It implies that the Sequence 2.1 is left quasi-exact, which is $D$-exact in $C$.

Consider now for any submodule $L$ of $B$, a left quasi-exact sequence

$$
0 \longrightarrow A \stackrel{f}{\longrightarrow} B \stackrel{g}{\longrightarrow} C \stackrel{h}{\longrightarrow} D
$$

induces another left quasi-exact sequence, as we give in the following proposition.

Proposition 2.4. Let $0 \longrightarrow A \stackrel{f}{\longrightarrow} B \stackrel{g}{\longrightarrow} C \stackrel{h}{\longrightarrow} D$ be a left quasi-exact sequence, $L$ be a submodule of $B$. If $M=g(L), K=f^{-1}(f(A) \cap L), N=h g(L)$, $f_{1}=\left.f\right|_{K}, g_{1}=\left.g\right|_{L}$, and $h_{1}=\left.h\right|_{M}$, then the sequence

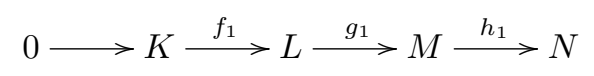

is left quasi-exact.

Proof. Based on Definition 2.1, $f$ is injective and $f_{1}=\left.f\right|_{K}$ is injective. Moreover, $\operatorname{Im}(f)=g^{-1}\left(U_{C}\right)$ and $\operatorname{Im}(g)=h^{-1}\left(U_{D}\right)$ for submodules $U_{C}$ in $C$ and $U_{D}$ in $D$. 
Now take $X=g\left(g^{-1}\left(U_{C}\right) \cap L\right)$ and we want to show that $\operatorname{Im}\left(f_{1}\right)=g_{1}{ }^{-1}(X)$. For any $b \in \operatorname{Im}\left(f_{1}\right)$, we have $b=f_{1}(a)=f(a)$ for some $a \in K$ such that $b \in f(A) \cap L$. Since $f(A)=\operatorname{Im}(f)=g^{-1}\left(U_{C}\right), b \in g^{-1}\left(U_{C}\right) \cap L$ such that $g_{1}(b)=g(b) \in X$. Thus $b \in g_{1}^{-1}(X)$ and $\operatorname{Im}\left(f_{1}\right) \subseteq g_{1}^{-1}(X)$. Conversely, for any $b \in g_{1}{ }^{-1}(X)$, we have $b \in g^{-1}\left(U_{C}\right) \cap L$. Since $g^{-1}\left(U_{C}\right)=\operatorname{Im}(f)=f(A), b \in f(A) \cap L$ and $b=f(a)$ for some $a \in A$. There exists $a \in f^{-1}(f(A) \cap L)=K$, where $f_{1}(a)=f(a)=b$ such that $b \in \operatorname{Im}\left(f_{1}\right)$. Hence $g_{1}{ }^{-1}(X) \subseteq \operatorname{Im}\left(f_{1}\right)$ and we prove that $\operatorname{Im}\left(f_{1}\right)=g_{1}{ }^{-1}(X)$.

Take $Y=h\left(h^{-1}\left(U_{D}\right) \cap M\right)$ and we want to show that $\operatorname{Im}\left(g_{1}\right)=h_{1}^{-1}(Y)$. For any $c \in \operatorname{Im}\left(g_{1}\right)$, it is clear that $c \in \operatorname{Im}\left(g_{1}\right) \subseteq \operatorname{Im}(g)=h^{-1}\left(U_{D}\right)$. It implies $c=g_{1}(b)=g(b)$ for an element $b \in L$ such that $c \in g(L)=M$. It means $c \in h^{-1}\left(U_{D}\right) \cap M$, so $h_{1}(c)=h(c) \in Y$. Hence $c \in h_{1}{ }^{-1}(Y)$ and $\operatorname{Im}\left(g_{1}\right) \subseteq h_{1}{ }^{-1}(Y)$. Conversely, for any $c \in h_{1}{ }^{-1}(Y), c \in M=g(L)$ such that $c=g(b)$ for an element $b \in L$. It implies $c=g_{1}(b)$, such that $c \in \operatorname{Im}\left(g_{1}\right)$. Thus, $h_{1}{ }^{-1}(Y) \subseteq \operatorname{Im}\left(g_{1}\right)$ and $\operatorname{Im}\left(g_{1}\right)=h_{1}^{-1}(Y)$.

Let $A, B$ and $D$ be $R$-modules. $\operatorname{Hom}_{R}(D, A)$ is the set of all $R$-homomorphisms from $D$ to $A$. It is well known that $\operatorname{Hom}_{R}(D, A)$ is a commutative group and can be viewed as a $\mathbb{Z}$-module. Now we define $\mathbb{Z}$-homomorphism

$$
f_{*}: \operatorname{Hom}_{R}(D, A) \longrightarrow \operatorname{Hom}_{R}(D, B),
$$

where $f_{*}(\alpha)=f \alpha$ for any $\alpha \in \operatorname{Hom}_{R}(D, A)$. We prove a property of left quasiexact sequences related to the $\operatorname{Hom}_{R}(D, A)$.

Proposition 2.5. Let

$$
0 \longrightarrow A \stackrel{f}{\longrightarrow} B \stackrel{g}{\longrightarrow} C \stackrel{h}{\longrightarrow} D
$$

be a left quasi-exact sequence, which is $U_{C}$-exact in $B, U_{D}$-exact in $C$ and let $h$ be injective, where $U_{C}$ is a submodule of $C$ and $U_{D}$ is a submodule of $D$. Then for any $R$-module $M$, the sequence of $\mathbb{Z}$-modules

$$
0 \longrightarrow \operatorname{Hom}_{R}(M, A) \stackrel{f_{*}}{\longrightarrow} \operatorname{Hom}_{R}(M, B) \stackrel{g_{*}}{\longrightarrow} \operatorname{Hom}_{R}(M, C) \stackrel{h_{*}}{\longrightarrow} \operatorname{Hom}_{R}(M, D)
$$

is left quasi-exact, which is

$H U_{C}$-exact in $\operatorname{Hom}_{R}(M, B)$ and $H_{D}$-exact in $\operatorname{Hom}_{R}(M, C)$,

where

$H U_{C}=\left\{\rho: M \longrightarrow C \mid \operatorname{Im}(\rho) \subseteq U_{C}\right\}$ and $H U_{D}=\left\{\phi: M \longrightarrow D \mid \operatorname{Im}(\phi) \subseteq U_{D}\right\}$.

Proof. We prove that the Sequence 2.4 is $H U_{C}$-exact in $\operatorname{Hom}_{R}(M, B)$ and $H U_{D}$-exact in $H_{o m}(M, C)$ by showing that $f_{*}$ is injective, $\operatorname{Im}\left(f_{*}\right)=g_{*}^{-1}\left(H U_{C}\right)$ and $\operatorname{Im}\left(g_{*}\right)=h_{*}^{-1}\left(H U_{D}\right)$. 
Take any $\theta \in \operatorname{Ker}\left(f_{*}\right)$. It implies for any $x \in M, f \theta(x)=0$. Since $f$ is injective, we conclude that $\theta(x)=0$ for any $x \in M$ such that $\theta=0$. Hence $\operatorname{Ker}\left(f_{*}\right)=0$ and $f_{*}$ is injective.

Moreover, we want to show that $\operatorname{Im}\left(f_{*}\right)=g_{*}^{-1}\left(H U_{C}\right)$. Take any $\theta \in \operatorname{Im}\left(f_{*}\right)$. It means $\theta=f_{*}(\varphi)=f \varphi$ for a homomorphism $\varphi \in H_{R} m_{R}(M, A)$ such that for any $x \in M$ it hold $\theta(x)=f(\varphi(x))$ and moreover $\theta(x) \in \operatorname{Im}(f)$. The Sequence (2.3) is $U_{C}$-exact in $B$, so $\operatorname{Im}(f)=g^{-1}\left(U_{C}\right)$ such that $\theta(x) \in g^{-1}\left(U_{C}\right)$. Furthermore, $g \theta(x) \in U_{C}$ and $g \theta=g_{*}(\theta) \in H U_{C}$ such that $\theta \in g_{*}^{-1}\left(H U_{C}\right)$. Hence $\operatorname{Im}\left(f_{*}\right) \subseteq$ $g_{*}^{-1}\left(H U_{C}\right)$.

Conversely, take any $\gamma \in g_{*}^{-1}\left(H U_{C}\right)$. We obtain $g_{*}(\gamma)=g \gamma \in H U_{C}$, so $g \gamma(x) \in$ $U_{C}$ for any $x \in M$. It implies for any $x \in M, \gamma(x) \in g^{-1}\left(U_{C}\right)$. The Sequence (2.3) is $U_{C}$-exact in $B$, thus $\gamma(x) \in \operatorname{Im}(f)$. Since $f$ is injective, we can form a homomorphism $f^{-1}: \operatorname{Im}(f) \longrightarrow A$.

Consider now $D \stackrel{\gamma}{\longrightarrow} \operatorname{Im}(f) \stackrel{f^{-1}}{\longrightarrow} A$. We define a homomorphism $h: D \longrightarrow A$ where $h=f^{-1} \gamma$. As a consequence we have

$$
f_{*}(h)=f h=f f^{-1} \gamma=\gamma
$$

such that $\gamma \in \operatorname{Im}\left(f_{*}\right)$. Hence $g_{*}^{-1}\left(H U_{C}\right) \subseteq \operatorname{Im}\left(f_{*}\right)$. and $\operatorname{Im}\left(f_{*}\right)=g_{*}^{-1}\left(H U_{C}\right)$.

In a similar way we can also prove $\operatorname{Im}\left(g_{*}\right)=h_{*}^{-1}\left(H U_{D}\right)$. Hence, the Sequence (2.4) is $H U_{C^{-}}$exact in $H_{o m}(M, B)$ and $H U_{D^{-}}$-exact in $H_{R} m_{R}(M, D)$.

The converse of Proposition 2.5 is also true as we give below:

Proposition 2.6. Let

$$
0 \longrightarrow A \stackrel{f}{\longrightarrow} B \stackrel{g}{\longrightarrow} C \stackrel{h}{\longrightarrow} D
$$

be a sequence of $R$-modules, where $U_{C}$ is a submodule in $C$ and $U_{D}$ is a submodule in $D$. If for any $R$-module $M$, the sequence

$$
0 \longrightarrow \operatorname{Hom}_{R}(M, A) \stackrel{f_{*}}{\longrightarrow} \operatorname{Hom}_{R}(M, B) \stackrel{g_{*}}{\longrightarrow} \operatorname{Hom}_{R}(M, C) \stackrel{h_{*}}{\longrightarrow} \operatorname{Hom}_{R}(M, D)
$$

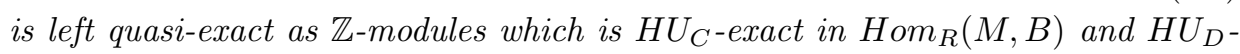
exact in $\mathrm{Hom}_{R}(M, C)$, where

$H U_{C}=\left\{\rho: M \longrightarrow C \mid \operatorname{Im}(\rho) \subseteq U_{C}\right\}$ dan $H U_{D}=\left\{\phi: M \longrightarrow D \mid \operatorname{Im}(\phi) \subseteq U_{D}\right\}$

then the Sequence 2.5 is left quasi-exact.

Proof. Take $M=\operatorname{Ker}(f)$, such that there is an inclusion homomorphism $i \in$ $\operatorname{Hom}_{R}(M, A)$. Moreover, $f_{*}(i)(\operatorname{Ker}(f))=f(\operatorname{Ker} f)=0$. Since $f_{*}(i)$ is injective, $\operatorname{Ker}(f)=0$, or equivalently, $f$ is injective. 
Now we choose $M=A$. Since the Sequence (2.6) is $H U_{C}$-exact in $H_{0}(M, B)$, we have $g_{*} f_{*}\left(1_{A}\right) \in H U_{C}$. We consider that $g_{*} f_{*}\left(1_{A}\right)=g_{*} f\left(1_{A}\right)=g f$, so for any $x \in A, g f(x) \in U_{C}$. It implies $f(x) \in g^{-1}\left(U_{C}\right)$ for any $x \in A$ such that $\operatorname{Im}(f) \subseteq g^{-1}\left(U_{C}\right)$. Conversely, take $M=g^{-1}\left(U_{C}\right)$. Then the mapping $j: M \longrightarrow B$ is an inclusion homomorphism and $g j(x) \in U_{C}$ for any $x \in M$. As a consequence, $g_{*}(j) \in H U_{C}$, such that $j \in g_{*}^{-1}\left(H U_{C}\right)$. The Sequence (2.6) is $H U_{C}$-exact in $H_{\text {omm }}(M, B)$, so $j \in \operatorname{Im}\left(f_{*}\right)$. It means $j=f_{*}(\alpha)=f \alpha$ for some $\alpha \in \operatorname{Hom}_{R}(M, A)$. Then for any $x \in M$ hold $x=j(x)=f \alpha(x) \in \operatorname{Im}(f)$ such that $M \subseteq \operatorname{Im}(f)$. Hence $g^{-1}\left(U_{C}\right) \subseteq \operatorname{Im}(f)$, or $\operatorname{Im}(f)=g^{-1}\left(U_{C}\right)$.

Furthermore, we can prove $\operatorname{Im}(g) \subseteq h^{-1}\left(U_{D}\right)$ by choosing $M=B$ and $h^{-1}\left(U_{D}\right) \subseteq \operatorname{Im}(g)$ in a similar way. Hence the Sequence 2.5$)$ is left quasi-exact.

\section{RIGHT QUASI-EXACT SEQUENCES}

The important point to note here is the fact that left quasi-exact sequences can be dualized into right quasi-coexact sequences.

Definition 3.1. A sequence $0 \longrightarrow A \stackrel{f}{\longrightarrow} B \stackrel{g}{\longrightarrow} C \stackrel{h}{\longrightarrow} D \longrightarrow 0$ is called a right quasi-coexact sequence if the sequence is $V_{A}$-coexact in $B, V_{B}$-coexact in $C$ and $h$ is surjective, where $V_{A}$ is a submodule of $A$ and $V_{B}$ is a submodule of $B$.

Our next example demonstrate the existance of right quasi-coexact sequence.

Example 3.2. The sequence $\mathbb{Z}$-modules

$$
\mathbb{Z}_{16} \stackrel{\pi_{1}}{\longrightarrow} \mathbb{Z}_{8} \stackrel{\pi_{2}}{\longrightarrow} \mathbb{Z}_{4} \stackrel{\pi_{3}}{\longrightarrow} \mathbb{Z}_{2} \longrightarrow 0
$$

is a right quasi-coexact sequence, where $\pi_{i}$ is a canonical projection, $i=1,2,3$.

A more technical point of view of Definition 3.1 is following. A sequence

$$
0 \longrightarrow A \stackrel{f}{\longrightarrow} B \stackrel{g}{\longrightarrow} C \stackrel{h}{\longrightarrow} D \longrightarrow 0
$$

is right quasi-coexact if $h$ is surjective, $f\left(V_{A}\right)=\operatorname{Ker}(g)$ and $f\left(V_{B}\right)=\operatorname{Ker}(h)$ for submodules $V_{A}$ in $A$ and $V_{B}$ in $B$. Moreover, a short $V$-coexact

$$
0 \longrightarrow B \stackrel{g}{\longrightarrow} C \stackrel{h}{\longrightarrow} D \longrightarrow 0
$$

is a special case of a right quasi-coexact sequence, where $A=0$. Furthermore, since every short $U$-exact sequence can be viewed as a short $V$-coexact, the short $U$-exact sequence $0 \longrightarrow B \stackrel{g}{\longrightarrow} C \stackrel{h}{\longrightarrow} D \longrightarrow 0$ is also a special condition of right quasi-coexact sequences. 
Lemma 3.3. A sequence

$$
A \stackrel{f}{\longrightarrow} B \stackrel{g}{\longrightarrow} C \stackrel{h}{\longrightarrow} D \longrightarrow 0
$$

is right quasi-coexact which is 0 -coexact in $B$ if and only if the sequence

$$
0 \longrightarrow B \stackrel{g}{\longrightarrow} C \stackrel{h}{\longrightarrow} D \longrightarrow 0
$$

is a short $V_{B}$-coexact sequence.

Proof. $(\Rightarrow)$ Based on the assumption and Definition 3.1, $h$ is surjective, $f(0)=$ $\operatorname{Ker}(g)$, and $f\left(V_{B}\right)=\operatorname{Ker}(h)$ for some submodule $V_{B}$ in $B$. Moreover, $0=\operatorname{Ker}(g)$ such that $g$ is injective. Then we can form the Sequence (3.2) where $g$ is injective, $h$ is surjective and $g\left(V_{B}\right)=\operatorname{Ker}(h)$ for a submodule $V_{B}$ in $B$. Hence, these sequence is short $V_{B}$-coexact.

$(\Leftarrow)$ Based on the assumption $g$ is injective, $h$ is surjective and $f\left(V_{B}\right)=\operatorname{Ker}(h)$ for some submodule $V_{B}$ in $B$. Since $g$ is injective, $\operatorname{Ker}(g)=0$. For any $R$ homomorphism $f: A \longrightarrow B, f(0)=0=\operatorname{Ker}(g)$. It yields the Sequence (3.1) where $f(0)=\operatorname{Ker}(g), f\left(V_{B}\right)=\operatorname{Ker}(h)$ for some submodule $V_{B}$ in $B$ and $h$ is surjective. Hence the Sequence (3.1) is right quasi-coexact which is 0-coexact in $B$.

Acknowledgement. We thank to the reviewers for the useful comments and suggestions.

\section{REFERENCES}

[1] Adkins, W.A., Weintraub, S.H., Algebra: An Approach via Module Theory, Springer-Verlag New York, Inc., USA, 1992.

[2] Anvariyeh, S.M., Davvaz, B., U-Split-Exact Sequences, Far East J. Math. Sci.(FJMS) 4 (2) (2002), 209-219.

[3] Anvariyeh, S.M., Davvaz, B. On Quasi-Exact Sequences, Bull Korean Math. Soc. 42 (1) (2005), 149-155.

[4] Davvaz, B., Shabani Solt, H. A Generalization of Homological Algebra, J. Korean Math. Soc. 39 (6) (2002), 881-898.

[5] Davvaz, B., Parnian-Garamaleky, Y.A., A Note on Exact Sequences, Bull.Malays. Math. Sci. Soc. 22 (1) (1999), 53-56.

[6] Wisbauer, R., Grundlagen der Modul- und Ringtheorie: Ein Handbuch für Studium und Forschung, Verlag Reinhard Fischer, München, 1988.

Siti Muawanah

(Master Student) Department of Mathematics, Universitas Gadjah Mada sitimwnh5@gmail.com

INDAH EMILIA WiJAYANTI

Department of Mathematics, Universitas Gadjah Mada

ind_wijayanti@ugm.ac.id 